\title{
Changes in chemical cues of Melissococcus plutonius infected honey bee larvae
}

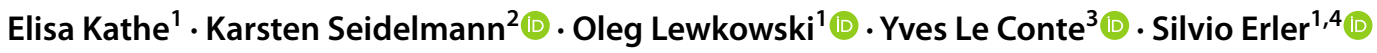

Received: 2 December 2020 / Accepted: 1 February 2021 / Published online: 18 February 2021

(c) The Author(s) 2021

\begin{abstract}
European foulbrood (EFB), caused by Melissococcus plutonius, is a globally distributed bacterial brood disease affecting Apis mellifera larvae. There is some evidence, even if under debate, that spreading of the disease within the colony is prevented by worker bees performing hygienic behaviour, including detection and removal of infected larvae. Olfactory cues (brood pheromones, signature mixtures, diagnostic substances) emitted by infected individuals may play a central role for hygienic bees to initiate the disease-specific behaviour. However, the mechanisms of cue detection and brood removal, causing hygienic behaviour in EFB affected colonies, are poorly understood. Here, coupled gas chromatography-mass spectrometry (GC-MS) was used to detect disease-specific substances, changes in cuticular hydrocarbon $(\mathrm{CHC})$ profiles, and brood ester pheromones (BEPs) of honey bee larvae artificially infected with M. plutonius. Although no diagnostic substances were found in significant quantities, discriminant analysis revealed specific differences in CHC and BEP profiles of infected and healthy larvae. $\beta$-Ocimene, a volatile brood pheromone related to starvation and hygienic behaviour, was present in all larvae with highest quantities in healthy young larvae; whereas oleic acid, a non-volatile necromone, was present only in old infected larvae. Furthermore, $\gamma$-octalactone (newly discovered in A. mellifera in this study) was detectable in trace amounts only in infected larvae. We propose that the deviation from the olfactory profile of healthy brood is supposed to trigger hygienic behaviour in worker bees. To confirm the relevance of change in the chemical bouquet (CHCs, BEPs, $\gamma$-octalactone, etc.), a field colony bioassay is needed, using healthy brood and hygienic bees to determine if bouquet changes elicit hygienic behaviour.
\end{abstract}

Keywords Apis mellifera $\cdot$ European foulbrood · Gas chromatography-mass spectrometry $\cdot$ Cuticular hydrocarbons · Brood ester pheromones $\cdot$ Hygienic behaviour

\section{Introduction}

Communicated by Marko Rohlfs.

Elisa Kathe and Karsten Seidelmann, authors contributed equally to this work.

Silvio Erler

silvio.erler@julius-kuehn.de

1 Institute of Biology, Molecular Ecology, MartinLuther-University Halle-Wittenberg, Hoher Weg 8, 06120 Halle (Saale), Germany

2 Institute of Biology, Animal Physiology, Martin-Luther-University Halle-Wittenberg, Hoher Weg 8, 06120 Halle (Saale), Germany

3 Abeilles Et Environnement, INRAE, 84914 Avignon, France

4 Institute for Bee Protection, Julius Kühn Institute (JKI)-Federal Research Centre for Cultivated Plants, Messeweg 11-12, 38104 Braunschweig, Germany
In the wake of the evolution of eusociality, hygienic behaviour has evolved as an important trait to control transmission and spread of diseases and parasites within social insect colonies (Cremer et al. 2007). In performing hygienic behaviour, worker honey bees, at the stage between nursing and foraging, detect and remove diseased larvae or pupae, even before they show visible symptoms (Evans and Spivak 2010). The two-step mechanism of hygienic behaviour includes not only the detection and removal of target individuals from open brood cells (bacteria and fungi-infected brood), but also from capped cells (mostly mite infested brood). The latter mechanism has an additional step, the uncapping of infested cells (removal of the wax lid) to get access to the brood and its parasite. The underlying mechanisms are based on olfactory cues to discriminate infected from healthy brood and to initiate brood removal. Both 
tasks are modulated by olfactory sensitivity (Evans and Spivak 2010; Gramacho and Spivak 2003; McAfee et al. 2017). Recent studies identified several hygienic behaviour associated volatile and non-volatile substances, emitted from freeze-killed brood (McAfee et al. 2017, 2018), brood infected with Ascosphaera spp. causing chalkbrood (Swanson et al. 2009) and brood infected with Paenibacillus larvae, the causative agent of American foulbrood (Lee et al. 2020). Honey bee brood infested with the ectoparasitic mite Varroa destructor (Martin et al. 2002; Mondet et al. 2016; Nazzi et al. 2004; Salvy et al. 2001; Wagoner et al. 2019) and brood parasitized by Varroa mites with high Deformed Wing Virus (DWV) loads (Schöning et al. 2012) also show infestation-specific bouquet profile changes, including abnormal BEP profiles (BEP-brood ester pheromones, a mixture of low-volatile fatty aliphatic esters) (Mondet et al. 2016). This shows that in the recent years specific substances as well as signature mixtures have been identified for most interactions of worker bees and diseased or dead offspring (e.g., cuticular hydrocarbons- $\mathrm{CHCs}\left(\mathrm{C}_{15}-\mathrm{C}_{36}\right)$, phenethyl acetate, 2-phenylethanol, benzyl alcohol, propionic acid, valeric acid, 2-nonanone, oleic acid, $E$ - $\beta$-ocimene, and brood ester pheromones) (Lee et al. 2020; Martin et al. 2002; McAfee et al. 2017, 2018, 2020; Mondet et al. 2016; Salvy et al. 2001; Schöning et al. 2012; Swanson et al. 2009; Wagoner et al. 2019). However, without showing any general pattern. In particular CHCs and BEPs are normally used by honey bees for communication within the community of the superorganism (e.g., kin, nestmate and brood recognition, fertility and reproduction, orientation, controlling feeding behaviour, etc.) (Blomquist and Bagnères 2010; Breed et al. 2015).

A largely neglected and understudied bacterial honey bee brood disease, inducing associated hygienic behaviour in worker bees, is European foulbrood (EFB). The Grampositive bacterium Melissococcus plutonius is considered to be the causative agent of EFB (Bailey 1956; White 1912, 1920). The lanceolate coccus multiplies in the midgut lumen of infected larvae and competes for nutrients leading to starvation and decomposition (Tarr 1937). The course of the disease is characterized by larval colour change from pearly white to yellow, brown, and at the final stage to greyish black, sometimes accompanied by a foul smell. Affected larvae are usually retarded in their development and stop feeding before ultimately dying (Forsgren 2010). However, not all infected larvae die after pathogen infection. Pathogen virulence depends on the M. plutonius genotype (Grossar et al. 2020; Lewkowski and Erler 2019). Several secondary invaders (e.g., Achromobacter eurydice, Bacillus pumilus, Brevibacillus laterosporus, Enterococcus faecalis, Paenibacillus alvei, Paenibacillus dendritiformis) are controversially discussed to increase the fatal effect (Bailey 1963; Erler et al. 2018; Forsgren 2010; Grossar et al. 2020; Lewkowski and Erler 2019). European foulbrood disease leads to substantial worldwide colony losses (Ellis and Munn 2005). Especially in England and Wales, as well as Switzerland, disease outbreaks have risen rapidly through the last decades (Roetschi et al. 2008; Wilkins et al. 2007; von Büren et al. 2019). Local variation in disease outbreaks has been observed, which might be due to a variability of strain virulence (Budge et al. 2014).

Bailey (1960) observed several colonies performing hygienic behaviour on 2- to 10-day-old infected honey bee larvae. Significantly more treated larvae were removed by worker bees before pupation than control larvae. In his study, healthy 1-day-old larvae were artificially infected with $M$. plutonius on brood combs and placed back inside healthy colonies. Moreover, Bailey assumed that in natural disease outbreaks larvae die before pupation and are ejected earlier compared to artificially infected larvae (Bailey 1960, 1963). His assumption might be based on the fact that naturally infected colonies show signs of malnutrition and may respond differently from artificially infected colonies.

This study focuses on odorant changes in EFB-diseased larvae that may serve as trigger for brood removal by hygienic worker bees. Honey bee larvae were artificially infected (Lewkowski and Erler 2019) using a highly virulent M. plutonius strain isolated in Switzerland (Djukic et al. 2018; Grossar et al. 2020; Lewkowski and Erler 2019). Chemical extracts and more precisely CHC profiles, BEP composition, oleic acid and $E$ - $\beta$-ocimene of in vitro reared larvae were characterized using coupled gas chromatography-mass spectrometry (GC-MS) to identify potential diagnostic substances and changes in the odorant bouquet related to the disease. The degree of the disease was characterized phenotypically by larval age and colour change. Healthy and diseased bees were further characterized for presence and quantity of $M$. plutonius. Finally, disease symptoms and olfactory signals were analysed for correlations that may show at which stage the disease might be detectable by the worker bees.

\section{Material and methods}

\section{Larvae rearing, bacteria cultivation and infection}

Apis mellifera worker larvae were reared in vitro according to the protocol of Crailsheim et al. (2013). A honey bee queen was caged on an empty brood frame for $24 \mathrm{~h}$. Three days after releasing the queen, freshly hatched worker larvae $(<24 \mathrm{~h})$ were grafted and transferred into 24 -well plates on $5 \mu$ day- 1 food solution, receiving food for 6 days (Crailsheim et al. 2013). To limit the variance of the larval bouquet (CHC profiles) to differences mainly resulting from the infection, only larvae of a single queen have been used for this study. 
Melissococcus plutonius (strain 49.3, wild type isolated in Switzerland, characterized in Djukic et al. 2018; Grossar et al. 2020) was cultivated in liquid medium (Lewkowski and Erler 2019) for 3 days, under semi-anaerobic conditions $\left(10 \% \mathrm{CO}_{2}\right)$. For larvae infection, bacteria-inoculated medium was mixed with day-1 food solution (Crailsheim et al. 2013) in a 1:10 ratio. The bacterial solution was previously adjusted to $\mathrm{OD}_{600 \mathrm{~nm}}=0.3$. Effective number of colony-forming units (CFU) was estimated by CFU counting using serial dilutions $(1: 10,1: 100,1: 1000$; Lewkowski and Erler 2019). The non-infected control group received day-1 food solution mixed with sterile culture medium at the same ratio. $5 \mu \mathrm{l}$ of the infective food mixture was fed to 1-day-old worker larvae right after grafting (final infective dose: $\sim 7.2 \times 10^{3}$ CFUs per larvae). Eight days post-grafting, all larvae were weighed and transferred into new 24-well plates for pupation (Lewkowski and Erler 2019). For survival analysis dead individuals (larvae, pupae, pre-adult bees) were counted daily until eclosion. Infection success and potential pathogen variance among samples (larval age, larvae grouped to different colour classes) was tested using M. plutonius specific qPCR with an internal A. mellifera reference gene (COI) for normalisation (Lewkowski and Erler 2019), and with the primer set of Budge et al. (2010) (EFBFor-TGTTGTTAGAGAAGAATAGGGGAA, EFBRev2-CGTGGCTTTCTGGTTAGA). Genomic DNA of M. plutonius strain 49.3 was used as positive control, as well as a non-template water sample for the negative control.

\section{Sampling and gas chromatography-mass spectrometry (GC-MS)}

Larvae of different age (day 5, 8, 9, 10 and 15 post-grafting and infection) and colour (white, yellow, brown, black, Fig. 1) were sampled daily, starting with day 5 post-grafting (Table S1). As for infected larvae, larval age and diseaseresulting colour change are partially correlated (on average older larvae show darker colour), not every time point was sampled with every larval colour (Table S1). Each 'infected larvae' replicate $(n=1-3)$ included three pooled larvae of the same age and colour in one glass vial according to the colour scheme shown in Fig. 1. At every time point, sampling diseased larvae, three replicates (with three larvae per replicate) of 'non-infected control larvae' were sampled as well. On day 8, after transferring larvae for pupation, remaining food, not consumed by the larvae, was pooled (due to low volume) and collected separately for each treatment group (infected, $n=6$, control, $n=5$ ). Remaining food, that also included some larvae faeces, was sampled to measure odorants secreted by the pathogen (M. plutonius) or the honey bee larvae at the feeding stage. Larvae or food remains ( $100 \mu \mathrm{l}$, respectively, per food replicate) were transferred into columnar AR-glasses $(60 \times 16.75 \mathrm{~mm})$, covered with $200 \mu \mathrm{l} n$-hexane (95\%, AppliChem) and extracted under medium shaking conditions for $10 \mathrm{~min}$ at room temperature. Samples were stored at $-80{ }^{\circ} \mathrm{C}$ for minimum $24 \mathrm{~h}$ to prevent odorant evaporation, to generate an extract in equilibrium and to ensure sampling of the liquid extract phase (Seidelmann and Rolke 2019). Thereafter, $100 \mu \mathrm{l}$ hexane extract (cuticular or food extract) were transferred into short thread ND9 glass vials (Carl Roth, Germany) and stored again at $-80{ }^{\circ} \mathrm{C}$ until GC-MS analysis. The reduced volume was used to prevent solvent contamination with food particles or larvae tissue.

Samples were analysed by a Saturn 2100 GC-MS (CP 3900 GC, Saturn 2100 T ion trap MS, Saturn-MS Workstation V 6.20; Varian Inc., California) equipped with an 1177 split/splitless injector and a VF-5 ms capillary column ( $30 \mathrm{~m} \times 0.25 \mathrm{~mm} \times 0.25 \mu \mathrm{m})$. Helium was used as carrier gas throughout. Oven temperature was programmed according to target substances (see below). Sample volumes of $1 \mu \mathrm{l}$ were injected in splitless mode. Mass spectra were acquired using standard $70 \mathrm{eV}$ electron impact ionization mode, mass range of $40-400 \mathrm{~m} / \mathrm{z}$. Both larvae and food odorants were analysed to characterize $\mathrm{CHC}$-compounds. Low-boiling volatiles as BEP were furthermore analysed in selected high-content
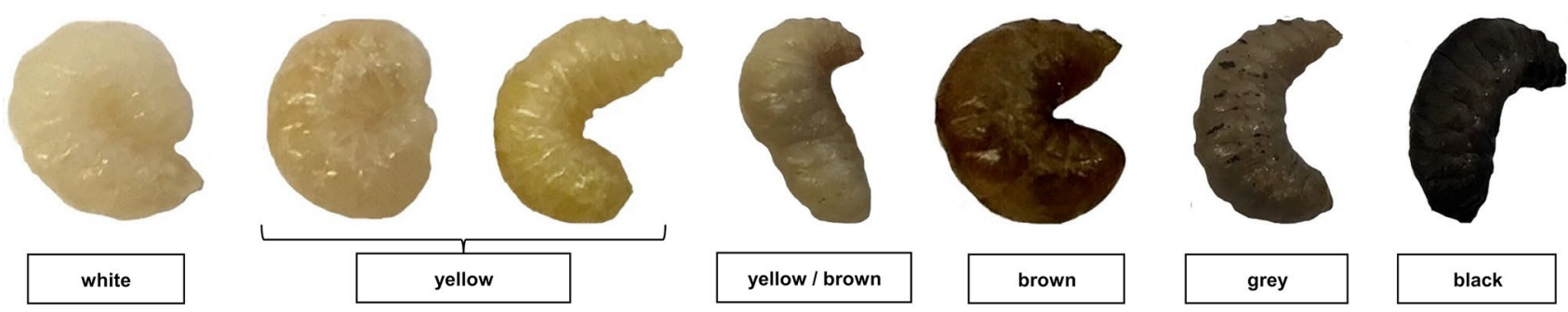

$8 \mathrm{~d}$

$9 \mathrm{~d}$

$10 \mathrm{~d}$

Fig. 1 Larval colour change during manifestation of the typical symptoms of EFB disease, from pearly white, yellow, brown to greyish black. Age (days post-grafting) of the larvae at each sampling point are marked with arrows. Three individuals of the same colour, according to this colour scheme, and same age were used for one GC-MS sample 
larvae samples. Substances have been identified by reference substances (e.g., alkanes $\mathrm{C}_{7}-\mathrm{C}_{40}$, BEP; Sigma-Aldrich) or their established substance-specific mass spectra (NIST MS-Database, Rev. 2.0) and those reported in the literature (Salvy et al. 2001), in conjunction with Kovats-indices determined using the alkane standard. For the identification of $\gamma$-octalactone (also known as 5-butyldihydro-2(3H)furanone; 4-hydroxy octanoic acid; octano-1,4-lactone; $\gamma$-octanoic lactone; CAS 104-50-7), a synthetic reference substance (Sigma-Aldrich) was used.

Cuticular hydrocarbon profiles: $\mathrm{CHC}$-compounds were identified and analysed according to the checklist and data provided by Salvy et al. (2001). GC was programmed to a linear temperature gradient of $10^{\circ} \mathrm{C} / \mathrm{min}$, from 80 to $320^{\circ} \mathrm{C}$, $4 \mathrm{~min}$ at $320^{\circ} \mathrm{C}$, constant flow rate of $0.7 \mathrm{ml} / \mathrm{min}$, and injector temperature $250{ }^{\circ} \mathrm{C}$. Mass fragments were detected from 6 to $30 \mathrm{~min}$.

Brood ester pheromone (BEP) composition and diagnostic substance: BEP profiles of honey bee larvae typically include methyl and ethyl esters of palmitic, stearic, oleic, linoleic and linolenic acid (Le Conte et al. 1989, 1990). Oleic acid $\left(\mathrm{C}_{18} \mathrm{H}_{34} \mathrm{O}_{2}\right)$ and $(E)$ - $\beta$-ocimene $\left(\mathrm{C}_{10} \mathrm{H}_{16}\right)$ have been discovered to be related to larval food-begging, larval starvation and brood removal by worker honey bees (He et al. 2016; Maisonnasse et al. 2010; McAfee et al. 2017, 2018). Under normal colony conditions, starving honey bee larvae emit higher amounts of the volatile brood pheromone $\beta$-ocimene and thus modulate the behaviour of worker bees to visit cells more frequently (He et al. 2016). $\beta$-Ocimene is also a major compound of the odour bouquet released by 0-3-dayold larvae, whereas 5-day-old larvae produce more of the low-volatile brood ester pheromones (BEPs) and vice versa (Maisonnasse et al. 2010). To quantify these lower boiling substances, the temperature program started at $40{ }^{\circ} \mathrm{C}$ with a linear gradient of $10{ }^{\circ} \mathrm{C} / \mathrm{min}$ to $300{ }^{\circ} \mathrm{C}$, injector temperature $240{ }^{\circ} \mathrm{C}$, flow rate $1 \mathrm{ml} / \mathrm{min}$, detection window of 5-30 min. Methyl linoleate was not detectable in any sample, and ethyl linoleate was detectable in a few samples, however not in quantifiable amounts (Fig. S1). Both compounds were omitted from further analysis. Methyl palmitate was measurable only in $36 \%$ of the samples in reliable quantities and had to be removed from the final data set as well.

\section{Data analysis}

Larval mortality was compared using Kaplan-Meier survival analysis following log-rank test. Larval weight and bacterial loads (M. plutonius qPCR) were tested by Shapiro-Wilk test for normal distribution, which could not be confirmed. Mann-Whitney $U$ tests and Kruskal-Wallis ANOVAs (following Bonferroni adjusted Dunn's post hoc test) were used to test for significant weight differences (mean $\pm \mathrm{SD}$ ) between infected and control larvae, as well as for variance in $M$. plutonius bacterial loads among samples. All data were analysed using Statistica 8.0 (StatSoft) and R (version 3.4.2).

Cuticular hydrocarbon GC-MS data (peak area and corresponding retention time), generated by automatic integration, were manually verified with due regard to mass spectra and exact retention times and manually re-integrated in case of a deviation. Some compounds occurred randomly in few samples only in very low quantities and have been excluded from further analysis. Relevant compounds were checked for randomly (not age- or treatment-specific) distributed zero values and were excluded to generate a reliable dataset. The final dataset consisted of 32 compounds for each of the 25 larval samples. Peak areas of these compounds were converted by quantile normalization ('preprocessCore' package, Bolstad 2016; R Core Team 2017) and centred log-ratio transformation (Seidelmann and Rolke 2019) to achieve normal distribution. The R 'stats' package (R Core Team 2017) was used for performing a Principle Component Analysis (PCA). A discriminant function analysis was run based on all principle components, with eigenvalues above 1 , to compare and visualize changes in the composition of cuticular hydrocarbon profiles of $M$. plutonius-infected and control larvae. Comparing the food samples, many substances could not be detected reliably and were listed as 'zeros'. Consequently, a quantile normalization was not possible and substance raw peak area data were used for subsequent analysis. Four substances of the larvae data set could not be detected in food samples and were eliminated from the final food data set. Raw data of honey bee larvae BEP compounds were checked, normalized, transformed and analysed (PCA and discriminant function analysis) as described for the cuticular hydrocarbons.

Characteristic bouquet compounds were normalized to the pentacosane and heptacosane peak area (acting as internal standards), the two $\left(n-\mathrm{C}_{25}, n-\mathrm{C}_{27}\right)$ major compounds of the honey bee larvae profile (Salvy et al. 2001). Normalized values were used to test for significant differences between age groups and/or larvae infected with $M$. plutonius vs. healthy, and to test for correlations between compound quantities, bacterial loads or age groups, respectively. Nonparametric tests were applied as data sets were not normally distributed.

\section{Results}

Melissococcus plutonius infection success was shown by treatment-dependent weight differences and larval survival. Infected individuals had a lower weight $(95 \pm 31 \mathrm{mg}$, $n=141)$ compared to controls $(141 \pm 20 \mathrm{mg}, n=131)$ (MWU test: $U=2142.5, p<0.0001)$. Larval mortality at day 21 post-grafting was higher for infected individuals $(76.5 \%$, 
$\left.n_{\text {all }}=119\right)$ than for the non-infected control group (18\%, $n_{\text {all }}=138$ ) (Kaplan-Meier survival, post hoc log-rank test: $\chi^{2}=9.15, p<0.0001$, Fig. S2). This means that $23.5 \%$ of the infected larvae survived the infection and developed into adult worker bees.

Nearly all infected larvae samples, used for GC-MS analysis, as well as the positive control were tested positive for M. plutonius using bacteria-specific qPCR. Only four individual larvae, as well as the negative control, were negative for M. plutonius ( 1 of 3 larvae of one day 8 yellow/brown colour replicate, all three larvae of the day 10 white colour replicate). Two larvae (one of three larvae of one day 8 white colour replicate- $\mathrm{Cq}=29.87$, one of three larvae of the day 10 yellow/brown colour replicate, $\mathrm{Cq}=29.88$ ) showed extremely low bacterial loads compared to the group with the highest loads $(0.04 \%$ of the median of day 5 infected larvae, $\mathrm{Cq}=18.15$ ). These two samples, with relative load values close to zero (white larvae: 0.0006 and yellow/brown larvae: 0.0001 ), were excluded from further M. plutonius load analysis. For all samples remaining, individual $M$. plutonius loads differed among larval age (Kruskal-Wallis ANOVA: $H=10.48, \mathrm{df}=4, n=39, p=0.033)$, with day 5 old larvae showing much higher values $(\mathrm{Cq}$ median $=18.15)$ than day 8 larvae $(\mathrm{Cq}$ median $=19.38$, which means a 3.73times higher load for day 5) $\left(z^{\prime}=2.86, p=0.042\right)$. All other time points did not differ from each other in $M$. plutonius loads. There was no significant interaction of larval colour (Fig. 1) and M. plutonius loads (Kruskal-Wallis ANOVA: $H=9.40, \mathrm{df}=4, n=39, p=0.052$ ), however a larger sample size should be used in future studies to test this potential interaction.

By comparing the bouquet profiles of healthy and infected larvae, a single volatile compound identified as $\gamma$-octalactone showed to be present nearly exclusively in infected larvae (MWU test: $U=15, n=25, p=0.002$ ) (Table S2). Among infected samples, $\gamma$-octalactone quantities increased with increasing infection loads (Spearman rank correlation, $r=0.64, p<0.01$ ), but not with larval colour change (Spearman rank correlation, $r=0.06$ ).

GC-MS data of larval extracts revealed that the main compounds of the larval cuticular hydrocarbon bouquet were saturated, branched and methyl-alkanes as well as alkenes (monoenes and dienes) (Table 1). Identified hydrocarbons ranged from chain lengths $\mathrm{C}_{17}-\mathrm{C}_{31}$. Cuticular hydrocarbon profiles of both treatment groups showed no differences in the identity of substances detected (Fig. 2). The PCA reduced the 32 components (peak no. 2-42) detectable in all control and infected individuals to seven factors explaining $85.36 \%$ of the total variance. The discriminant analysis separated larvae not only by age (day 5 vs. $\geq 8$ post-grafting; Factor 1: $34.5 \%$ of total variance, eigenvalue: 11.03 ), but also by treatment (infected vs. control; Factor 2: $15.4 \%$ of total variance, eigenvalue: 4.94$)$. The two factors represent about
$50 \%$ of the total variability of the dataset. Few infected individuals cluster together with non-infected controls (Fig. 3). Larvae may survive the infection as shown by $23.5 \%$ larvae surviving until day 21 post-grafting (Fig. S2). One larva of the samples of day 8 yellow/brown and day 10 yellow/brown showed to be non-infected or had very low M. plutonius loads. With showing Cq-values of approx. 30 (see above) we can only speculate that the larvae were infected (without corresponding disease symptoms), or the bacteria proliferated at very low frequency, or these values came from feeding infectious food and the bacteria did not replicate. These single larvae may exude an odour bouquet like healthy larvae, grouping the whole sample to this specific cluster.

The main compounds contributing to the positive part of the first dimension, discriminating honey bee larvae by age, are long-chained linear alkanes $\left(\mathrm{C}_{27}-\mathrm{C}_{29}\right.$, Table 1, Fig. S3). Whereas shorter (methyl-branched) alkanes represent the negative part of the first dimension (Table 1, Fig. 3, Fig. S3). Two CHC compounds strongly decreased with increasing larval age (Spearman rank correlation, $\mathrm{C}_{22}$-docosane: $r=-0.72, \mathrm{C}_{25}$-pentacosane: $r=-0.93$, both $p<0.001$ ). The positive and negative part of the second dimension, discriminating the samples by treatment, was almost exclusively driven by odd (methyl-branched) alkanes and alkenes $\left(\mathrm{C}_{19}-\mathrm{C}_{31}\right)$ (Table 1, Fig. 3, Fig. S3). For the food samples, the PCA reduced the 39 components to four factors explaining $93.41 \%$ of the total variance. The discriminant analysis separated food samples by treatment, with $56.8 \%$ (Factor 1, eigenvalue: 22.15) of the total variance (Fig. S4), albeit a high variance among non-infected control food samples can be observed.

The PCA for the seven BEP compounds reduced all components to three factors explaining $82.22 \%$ of the total variance. The discriminant analysis revealed one narrow group of old infected larvae ( $\geq$ day 8 ), surrounded by healthy old ( $\geq$ day 8 ) and healthy and infected young (day 5) larvae (Fig. 4). The first two factors explain $66.6 \%$ of the total variance (Factor 1, eigenvalue: 3.28; Factor 2, eigenvalue: 1.38). The clustering of the old infected larvae was mainly driven by ethyl oleate, ethyl palmitate and ethyl stearate (Fig. S5, Table S2). Comparing the different age groups showed that methyl oleate and methyl linolenate were much higher in younger (day 5) than in older ( $\geq$ day 8) larvae (MWU test: $U_{\text {methyl oleate }}=22.5, U_{\text {methyl linolenate }}=23.5, n_{\text {each }}=25$, $p<0.05)$, whereas methyl stearate and ethyl stearate were much higher in older ( $\geq$ day 8 ) than in young larvae (day 5) (MWU test: $U_{\text {methyl stearate }}=25.5, U_{\text {ethyl stearate }}=6, n_{\text {each }}=25$, $p<0.05$ ) (Table S2).

The brood pheromone $\beta$-ocimene was present in all larvae samples, with younger larvae (day 5) showing much higher values than older (all larvae $\geq$ day 8 ) (MWU test: $U=6$, $p=0.0013$ ) (Table S2). Infection with M. plutonius decreased $\beta$-ocimene compared to healthy controls (MWU test: $U=30$, 
Table 1 Main cuticular hydrocarbon profile compounds identified by GC-MS with peak number, compound name, retention time, and calculated retention indices (Lee et al. 1979). Results are given for the

\begin{tabular}{|c|c|c|c|c|c|}
\hline Peak no. & Compound name & $\begin{array}{l}\text { Retention time } \\
(\mathrm{min})\end{array}$ & Retention index & Factor 1 & Factor 2 \\
\hline 2 & $n-\mathrm{C} 17: 0$ & 14.294 & 1700 & -0.238 & 0.060 \\
\hline 3 & Unknown & 14.383 & 1707 & -0.255 & -0.034 \\
\hline 5 & $n-\mathrm{C} 19: 0$ & 16.460 & 1900 & -0.018 & -0.283 \\
\hline 7 & 9-C21:1 & 18.162 & 2074 & 0.078 & 0.109 \\
\hline 8 & $\mathrm{C} 21: 0$ & 18.397 & 2100 & 0.145 & -0.212 \\
\hline 9 & Unknown & 19.015 & 2166 & 0.243 & -0.091 \\
\hline 11 & $n-\mathrm{C} 22: 0$ & 19.313 & 2200 & -0.109 & 0.004 \\
\hline 12 & 9-C23:1 & 19.970 & 2274 & -0.075 & -0.333 \\
\hline 13 & $n-\mathrm{C} 23: 0$ & 20.192 & 2300 & -0.081 & -0.325 \\
\hline 14 & $11+9-\mathrm{MeC} 23$ & 20.484 & 2334 & -0.258 & 0.011 \\
\hline 15 & 7,17-diMeC23 & 20.812 & 2373 & -0.277 & 0.056 \\
\hline 16 & $n-\mathrm{C} 24: 0$ & 21.013 & 2400 & 0.057 & 0.064 \\
\hline 17 & 9-C25:1 & 21.650 & 2476 & -0.255 & -0.116 \\
\hline 18 & $n-\mathrm{C} 25: 0$ & 21.842 & 2500 & -0.225 & -0.081 \\
\hline 19 & $13+11+9+7-\mathrm{MeC} 25$ & 22.097 & 2533 & -0.215 & 0.162 \\
\hline 20 & $5-\mathrm{MeC} 25$ & 22.228 & 2549 & -0.145 & 0.199 \\
\hline 21 & 4-MeC25 +9,17-diMeC25 & 22.327 & 2562 & 0.172 & 0.180 \\
\hline 22 & 3-MeC25 + 7,15-diMeC25 + 7,17-diMeC25 & 22.391 & 2570 & 0.155 & 0.290 \\
\hline 23 & 5,15-diMeC25 & 22.487 & 2583 & -0.184 & 0.078 \\
\hline 25 & $\mathrm{C} 27: 2+14-\mathrm{MeC} 26$ & 22.855 & 2631 & 0.104 & 0.231 \\
\hline 26 & 9-C27:1 + x,y-diMeC26 & 23.186 & 2675 & -0.041 & -0.109 \\
\hline 27 & $n-\mathrm{C} 27: 0$ & 23.373 & 2700 & 0.283 & 0.004 \\
\hline 28 & $15+13+11+9+7+5-\mathrm{MeC} 27$ & 23.584 & 2731 & -0.069 & 0.189 \\
\hline 29 & 11,17-diMeC27 + 11,15-diMeC27 +9,x-diMeC27 & 23.774 & 2756 & -0.058 & 0.360 \\
\hline 32 & $n-\mathrm{C} 28: 0$ & 24.083 & 2800 & 0.259 & 0.031 \\
\hline 33 & $14+13+12+11-\mathrm{MeC} 28$ & 24.295 & 2829 & 0.193 & 0.182 \\
\hline 34 & 9-C29:1 + C29:2 + 2-MeC28 & 24.634 & 2877 & 0.204 & -0.213 \\
\hline 35 & $n-\mathrm{C} 29: 0$ & 24.793 & 2900 & 0.261 & -0.047 \\
\hline 36 & $15+30+11+9+7-\mathrm{MeC} 29$ & 25.002 & 2931 & 0.018 & 0.098 \\
\hline 40 & 9-C31:1 & 25.992 & 3080 & -0.018 & -0.244 \\
\hline 41 & $n-\mathrm{C} 31: 0$ & 26.111 & 3100 & 0.187 & -0.125 \\
\hline 42 & $15+13+11-\mathrm{MeC} 31$ & 26.305 & 3127 & -0.026 & 0.146 \\
\hline
\end{tabular}

$p=0.019$ ) (Table S2), with no correlation between $\beta$-ocimene and $M$. plutonius loads for infected larvae (Spearman rank correlation, $r=0.22$ ). Oleic acid was detectable only in larvae older than day 5 and infected with M. plutonius (Table S2), however without any significant differences between age groups (day 8-15, Kruskal-Wallis ANOVA: $H=8.56$, $\mathrm{df}=4$, $n=16, p=0.07$ ) or any correlation with $M$. plutonius loads (Spearman rank correlation, $r=0.12$ ). first two factors of the discriminant analysis describing $~ 50 \%$ of the total variance (Factor 1 and 2). (Branching points are given according to the data from Salvy et al. 2001.)

\section{Discussion}

Artificial infection of in vitro reared honey bee larvae generated phenotypes that were highly suitable to study differences in chemical bouquet composition of A. mellifera larvae. The following parameter (survival, colour, weight) made it possible to describe and group larval 


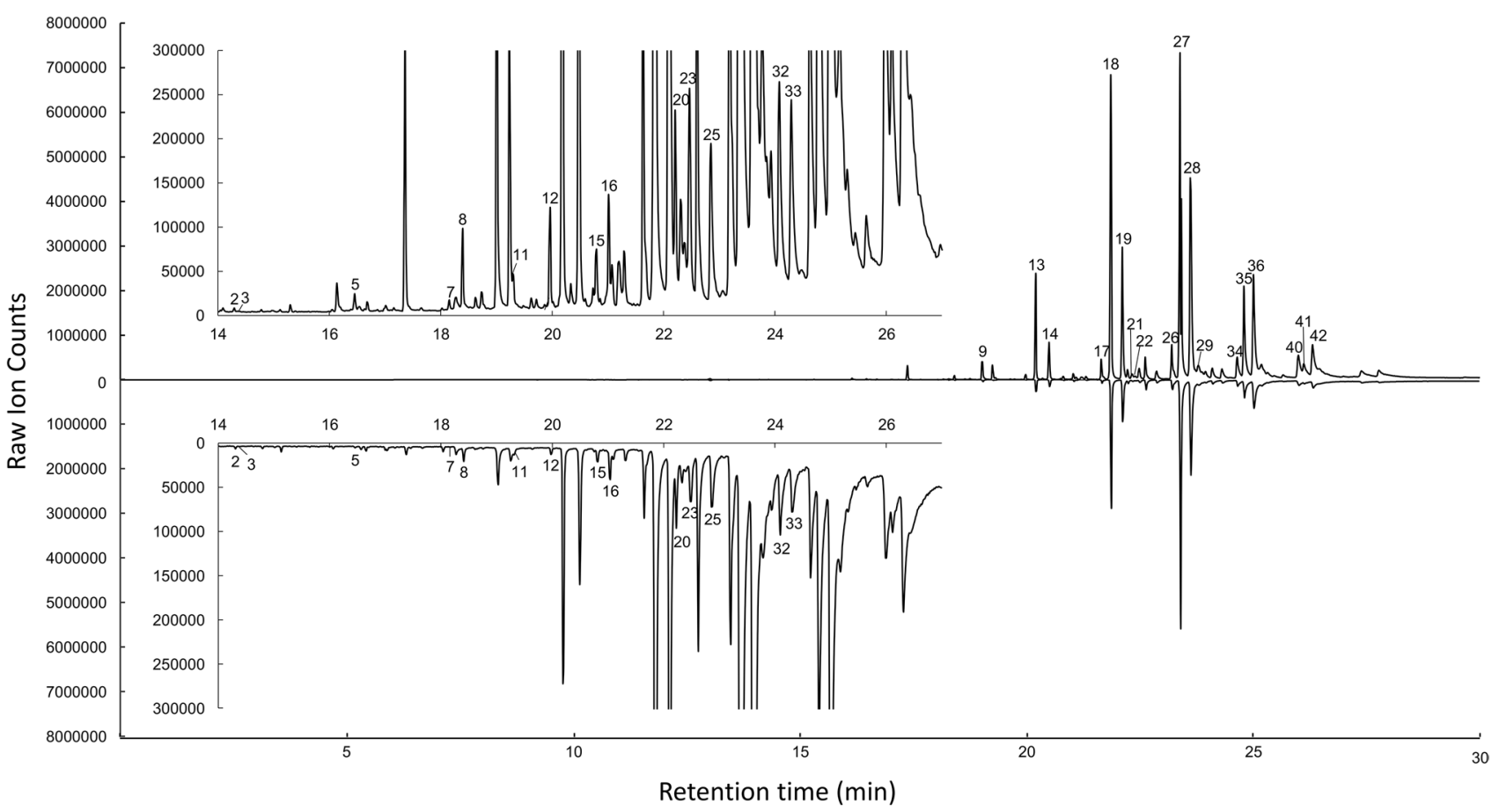

Fig. 2 Representative cuticular hydrocarbon profiles of 8-day-old infected (upper panel) and non-infected (lower panel) honey bee larvae analysed by GC-MS. PCA-relevant peaks are labelled according to Table 1. Peaks with raw ion counts $<300,000$ are zoomed and shown separately

samples according to their phenotypic changes. The successful infection and resulting disease-specific phenotype was characterized by a high mortality rate of infected individuals until day 21 post-grafting; corresponding well with data of previous studies (Grossar et al. 2020; Lewkowski and Erler 2019; Nakamura et al. 2016; Riessberger-Gallé et al. 2016). The second parameter for monitoring the course of the disease was larval colour change (Fig. 1). Characteristic colour change made it possible to combine larvae to different 'groups' of clinical symptoms accompanied with EFB disease (Forsgren et al. 2013, 2018). Finally, all larvae that survived until day 8 showed strong differences in body weight between infected and control larvae. M. plutonius-infected larvae are usually lighter, compared to control individuals (Bailey 1960, 1963; Lewkowski and Erler 2019). This difference in larval weight is attributed to starvation (Tarr 1937), driven by the host-pathogen competition for nutrients. Under natural conditions, honey bee larvae receive their food from nurse worker bees by progressive feeding and worker bees modify food composition depending on larval developmental stage ( $\leq 3 \mathrm{~d}$ : royal jelly, $>3 \mathrm{~d}$ : worker jelly). This means worker larvae have limited access to food jelly, they do not 'float' on huge quantities of food jelly as they did under artificial rearing in the current study. The higher food demand, solicited from starving infected larvae, may not be compensated by higher feeding frequency of worker bees in the hive. Consequently, the weight of infected larvae might be reduced even stronger under natural conditions as symptom of the bacteria-caused malnutrition of the larvae (Bailey 1959, 1960; Lewkowski and Erler 2019; Nakamura et al. 2016). Nevertheless, larval weight can be used as valid criterion to characterize the course of the disease under controlled in vitro rearing conditions.

While colour, weight and survival are mostly visual parameters describing the disease, worker bees use olfactory cues for orientation and brood care inside the hive. Removing diseased brood is the second step of hygienic behaviour after successful identification and differentiation from healthy individuals. Olfactory marker substances or signature mixtures are well known to initiate hygienic behaviour. For example, Swanson and colleagues (2009) detected three specific compounds (phenethyl acetate, 2-phenylethanol and benzyl alcohol) in 5-day-old larvae infected by the fungal pathogen Ascosphaera apis (chalkbrood), and only one single compound (phenethyl acetate) induced hygienic behaviour on chalkbrood diseased individuals or paraffin larval dummies treated with phenethyl acetate. 2-Phenylethanol was detectable in trace quantities in $20 \%$ of our EFB-samples, however without any disease-specific pattern. Day 7 old honey bee larvae, infected with $P$. larvae (AFB pathogen), were characterized by several volatile disease markers (propionic acid, valeric acid, 2-nonanone) that can be detected by more sensitive middle-aged bees rather than by forager bees 


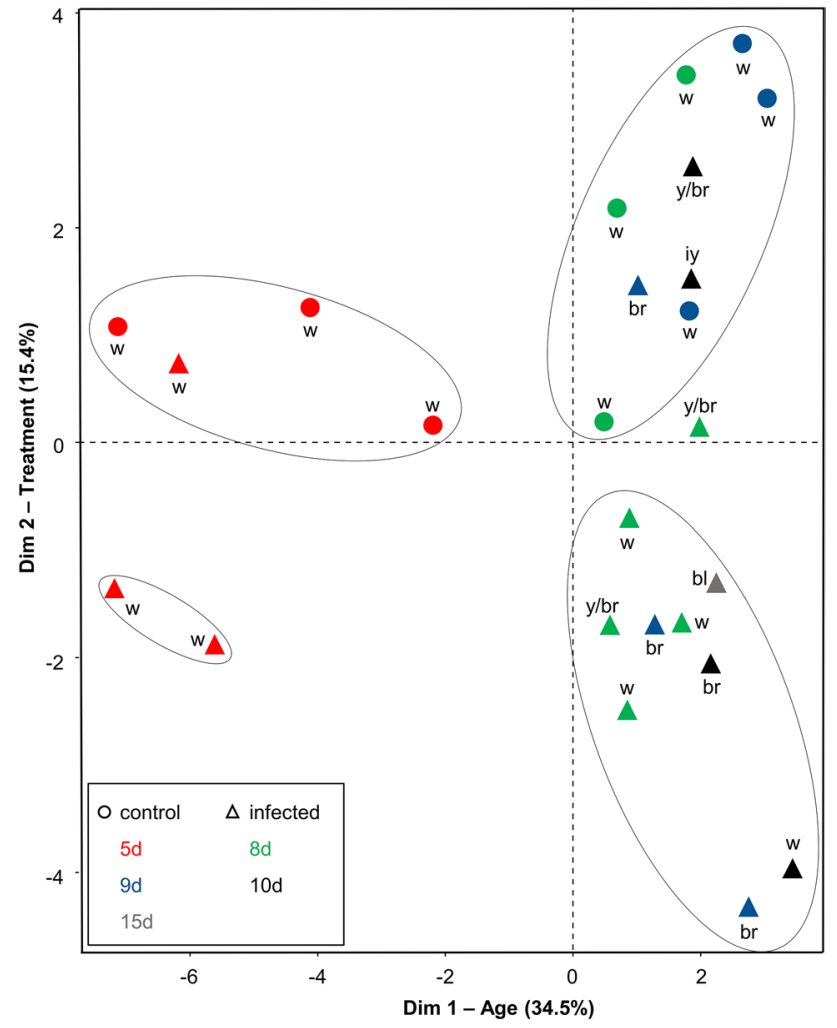

Fig. 3 Discriminant analysis of honey bee larvae cuticular hydrocarbon profiles separated by age ( 5 vs. $\geq 8$ days post-grafting) and treatment (infected $v s$. control), by the two first dimensions (49.9\% of the total variance). Sample labels contain larval age (day post-grafting), treatment (infected-triangles, control-circles) and colour ( $w$ white, $y$ yellow, $y / b r$ yellow/brown, $b r$ brown, $b l$ black) according to Table 1 and Fig. 1. Ellipses highlight distinct sample groups

(Lee et al. 2020). However, it is still elusive if and how they are involved in hygienic behaviour. Phenethyl acetate and the volatile diagnostic substances of American foulbrood disease were not present in diseased larvae of the current study. Benzyl alcohol and propionic acid were not detectable in M. plutonius-infected larvae as both run in parallel with the solvent peak due to their low Kovats-indices. Solid phase micro extraction (SPME) can be used in future studies to investigate highly volatile substances (e.g., $\mathrm{H}_{2} \mathrm{~S}$ ) that might also be involved in the eponymous foul smell of the brood. Currently, with the random presence of 2-phenylethanol we might conclude that the fungal disease chalkbrood and the bacterial diseases (AFB and EFB) do not share the same identification pattern for hygienic worker bees. Even volatile disease markers, specific for another bacterial brood disease (AFB), that have the potential to be chemical cues for worker bees, could not be detected for EFB-disease. Altogether, we see that each microbial infection results in very distinct chemical profiles and there is no general disease signal for sick honey bee larvae. However, we have to mention that most studies focused mainly on the identification of

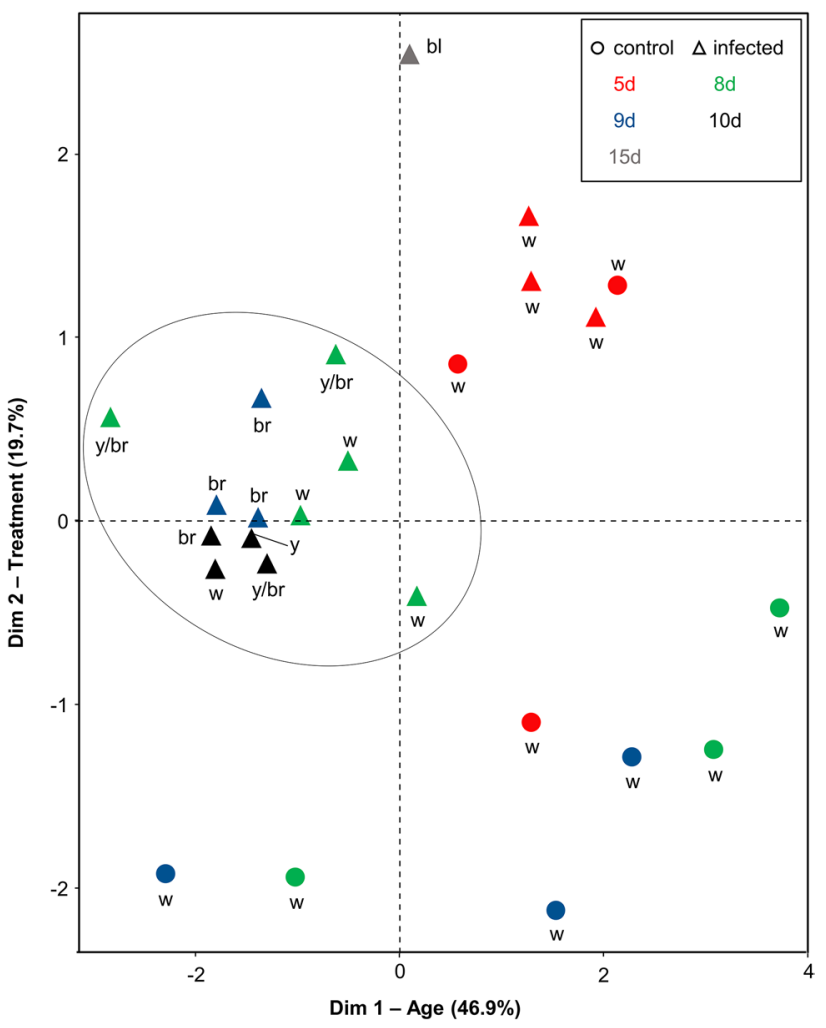

Fig. 4 Discriminant analysis of honey bee larvae brood pheromone profiles with a clear cluster (encircled) for older infected larvae, surrounded by older healthy and young (day 5) healthy and infected larvae. Sample labels contain larval age (day post-grafting), treatment (infected-triangels, control-circles) and colour ( $w$ white, $y$ yellow, $y / b r$ yellow/brown, $b r$ brown, $b l$ black) according to Fig. 1

disease-specific diagnostic substances. The identification of unique compound(s) in diseased brood is only the first step in determining biological relevance. Future studies should combine both, the characterization of the disease-associated identification pattern among the range of CHCs and BEPs, and the identification of putative signal substances. Hygienic bees may simply recognize the deviation from 'normal' by comparing the scent of healthy and diseased brood, which is the signal (pattern deviation) to eliminate 'abnormal' individuals. The innate scent of healthy brood can be expected to be learned based on the different tasks performed along the age polyethism during individual development of worker bees. In the case of EFB, worker bees might be even stricter when food resources become scanty.

Analysing CHC profiles, a relatively high proportion of the total variance, in addition to the first dimension (larval age), can be explained by the second dimension of the CHC discriminant analysis (Fig. 3); dividing the dataset into infected and uninfected individuals. M. plutonius infection caused a change in the composition of larval $\mathrm{CHC}$ profiles. The main compounds characterizing the treatment-dependent $\mathrm{CHC}$ change were odd hydrocarbons 
( $n$-alkanes, $n$-alkenes, and methyl-alkanes; peaks no. 5, 8, $12,13,22,25,29,34$ and 40) with a chain length of $\mathrm{C}_{19}$, $\mathrm{C}_{21}, \mathrm{C}_{23}, \mathrm{C}_{25}, \mathrm{C}_{27}, \mathrm{C}_{29}$ and $\mathrm{C}_{31}$ (Table 1, Fig. S3). In previous studies, quantitative changes in n-alkanes and alkenes with chains odd-numbered were likewise characteristic for Varroa destructor and Varroa jacobsoni infested honey bee pupae (Martin et al. 2001, 2002; Salvy et al. 2001; Wagoner et al. 2019). Changes in characteristic CHCs that are candidates for hygienic signalling were also stock-specific (Wagoner et al. 2019), therefore several hygienic lineages have to be tested to identify marker substances for hygienic selection. In the same context two substances have been identified recently, $(Z)$-6-pentadecene $\left(C_{15}\right)$ as biologically activity substance, and $(Z)$-10-tritriacontene $\left(\mathrm{C}_{33}\right)$ as a novel hygiene trigger (Wagoner et al. 2020). Varroa destructor infested (pre)-pupae further showed comparable results for BEP compounds with distinct BEP profiles for removed brood and differences in amounts of BEP components, in particular, of removed pupae (Mondet et al. 2016). Hygienic worker bees might use these disease-specific cues to identify infested brood cells (Mondet et al. 2016). In this study, older M. plutonius-infected larvae exuded a specific BEP bouquet, a potential chemical cue for brood removal. Once again, we want to stress our alternative hypothesis that hygienic workers may not use diagnostic substances or cues, they may simply remove everything that does not smell 'normal'. This seems plausible as worker bees do not need to 'learn' new substances for each disease; they only need to compare the odorant bouquet with that of normal, healthy brood. Potential mechanisms of diseased brood odorant bouquet comparison, in the olfactory lobes and the brain, against the excitation pattern of normal brood, and subsequent brood removal if patterns are non-matching, is still elusive and has to be tested in future experiments.

Brood pheromone blends, even single compounds, are well known to provide context-specific signals that induce different behaviours (Slessor et al. 2005). However, they need further investigation in the context of EFB disease, to understand their potential role for infected brood removal, as shown for Varroa-infested bees. Biotests with healthy or diseased brood/dummies, by manipulating BEP composition and quantities, might be a future project to unravel the actual signal for hygienic brood removal. However, it is mandatory to use different concentrations, as pheromone response ranges can be quite narrow and compound specific (Le Conte et al. 1995).

The CHCs discriminant analysis of this study, along with strong differences for $\beta$-ocimene, showed that $34.5 \%$ of the total variance was caused by age-dependent differences in the composition of volatile and non-volatile substances emitted by larvae. These age-related differences may act as cues modulating feeding and/or brood removal behaviour. On the contrary, age-related changes of CHCs, BEPs, and $\beta$-ocimene might be hard to differentiate from diseaserelated changes as the disease and its symptoms develop gradually over time. During development, the malnutrition of larvae as symptom of EFB disease might cause a higher emission of $\beta$-ocimene. Such a variation may be recognized by the nurse bees, in relation to a reference (e.g., BEP), and may trigger hygienic behaviour of workers. Here, under non-natural in vitro conditions, $\beta$-ocimene was detected in all larval stages with higher values for young individuals (day 5). It seems that the observed result is contrary to our expectation that older larvae show higher $\beta$-ocimene values than younger. However, the in vitro rearing may prevent the production of high quantities of $\beta$-ocimene. The artificial rearing protocol provides worker larvae with essential nutrients for completing their development, but their food consumption is limited for the 6-day feeding period (Crailsheim et al. 2013). This may resulted in the production of higher amounts of $\beta$-ocimene for the younger larvae to beg for additional food, based on the empty pharynx of the larvae. Infected larvae and larvae not completing their larval phase until day $7 / 8$ are expected to increase starvation signalling via $\beta$-ocimene production. Contrary to this expectation, infected as well as older (non-pupae) larvae produced lower quantities of $\beta$-ocimene, which might be explained by the presence of non-consumed food that accumulated over time. The availability of food will result in absence of signaling of an empty pharynx. Consequently, begging for additional food was not necessary. However, this mechanism will not prevent the inefficient uptake of nutrients in the gut system for larval metabolism. Finally, larvae floating on nonconsumed food will starve due to ineffective nutrient uptake even though they had enough food.

In natural EFB disease outbreaks, food limitation is of major importance. If food stores become empty and successful foraging does not ensure sufficient food availability in the bee hive, worker bees are supposed to eject more larvae (Bailey 1960). In this and other cases of insufficient food provisioning of larvae, $\beta$-ocimene is the relevant signal to communicate with the nurse bees. A potential reference substance or blend for the worker bees to estimate the signal strength of $\beta$-ocimene has to be discovered in future experiments. BEP, $\mathrm{C}_{25}$ or $\mathrm{C}_{27}$ quantities are potential candidates.

Infected older larvae ( $\geq$ day 8 ) were the only individuals in the current study that produced the necromone (death pheromone) oleic acid that is known as 'ejection' signal, which may induce infected brood removal. Various other studies showed that sick or dead larvae produce irregular high amounts of pheromones supposed to trigger hygienic behaviour. For instance, 12-17-day-old freeze killed pupae emitted significantly more $\beta$-ocimene and oleic acid (McAfee et al. 2017). Oleic acid has a central function for hygienic brood removal of Varroa-infested and dead pupae, and potentially for P. larvae infected brood (Lee et al. 2020; 
Martin et al. 2002; McAfee et al. 2017, 2018). However, if worker bees use oleic acid as signal to removed EFBinfected brood has to be confirmed by bioassay of future studies.

Comparing the chemical bouquet of healthy and diseased larvae, we discovered a so far unknown compound in the honey bee A. mellifera. M. plutonius-infected larvae extracts contained $\gamma$-octalactone, and quantities increased with increasing $M$. plutonius loads. So far, only few studies detected $\gamma$-octalactone (alias $\gamma$-octanoic lactone) in cephalic extracts from workers of A. laboriosa (Blum et al. 2000) and in the airspace around $A$. dorsata bees captured in vials $(\mathrm{Li}$ et al. 2014). The authors concluded that $\gamma$-octalactone might possess a specific pheromone that elicit significant alarm response (Blum et al. 2000; Li et al. 2014). $\gamma$-Octalactonespecific avoidance response has also been shown for $A$. cerana forager bees, for a compound that is not found in $A$. cerana alarm pheromone (Wang et al. 2016). This shows that $\gamma$-octalactone might also be produced by diseased honey bee larvae that may signal illness to workers and may induce hygienic behaviour. For future studies, $\gamma$-octalactone can be an ideal marker signal for diseased brood, even before visible signs can be detected. Nevertheless, the potential function of $\gamma$-octalactone for EFB disease-associated hygienic behaviour remains unknown.

Other cues for detecting M. plutonius infected larvae might originate from the bacterium itself, since microorganisms may produce taxa or even strain characteristic compounds like specific metabolites (Djukic et al. 2018; Lee et al. 2020). Bacteria derived disease markers (e.g., propionic and valeric acid) have recently been identified for $P$. larvae infected honey bee larvae (Lee et al. 2020). Different strains may also have various impact on larval odorant bouquet just as they have on larval mortality (Grossar et al. 2020; Lewkowski and Erler 2019; Nakamura et al. 2016). An analysis of the food samples revealed that there is a difference in composition of hydrocarbons between food remains of infected and control larvae, maybe caused by M. plutonius (Fig. S4). However, food samples were contaminated by larval faeces, which might have increased $M$. plutonius concentrations in samples of the infection group, leading to very distinct GC-MS profiles. Under natural hive conditions, further approach is given by secondary invaders, which are discussed to come along with natural disease outbreaks (Bailey 1963; Forsgren 2010; White 1920). They may also contribute to a significant change in the odorant bouquet to which worker bees respond with performing hygienic behaviour.

In conclusion, we demonstrated that $M$. plutonius infection-dependent changes of the odorant bouquet of artificially reared and infected $A$. mellifera larvae are detectable by GC-MS. The comparison of sick and healthy individuals revealed that $\gamma$-octalactone, brood ester pheromones, $\beta$-ocimene, oleic acid and other substances, mostly longchained hydrocarbons, are a good indication for an EFBspecific key for worker bees. These are prime candidates for EFB-associated hygienic behaviour, which does not match with key substances of other diseases, and clearly demonstrates that there is no unique signal to remove sick brood. The compounds identified in the current study might not only be relevant for EFB disease-specific brood removal, they have also been demonstrated to strongly act on honey bee worker behaviour in general (kin and nestmate recognition, brood recognition, controlling feeding behaviour, inhive orientation, etc.). However, a direct link between emitted EFB disease-associated chemical bouquets and hygienic behaviour is still pending. A field bioassay; i.e., application of $\gamma$-octalactone (and possibly in combination with other compounds such as BEPs, oleic acid and $\beta$-ocimene) to healthy brood in field colonies is needed to determine if the compounds elicit hygienic behaviour. Furthermore, a direct link between EFB disease and hygienic behaviour, especially in field, is still missing (only Bailey 1960) and has never been studied in natural disease outbreaks. Nevertheless, with the known function of olfactory signals for the honey bee superorganism, we hypothesize that the results of this study show to have an effect on the chemical interaction of honey bee brood, healthy or diseased, and hygienic workers bees.

Supplementary Information The online version contains supplementary material available at https://doi.org/10.1007/s00049-021-00339-3.

Acknowledgements We thank Jenifer Heidler and Juliane Fischer for help with lab work, Jean-Daniel Charrière (Swiss Bee Research Center, Agroscope) for providing the $M$. plutonius strain, and three anonymous reviewers for helpful suggestions and discussion on the manuscript.

Author's contribution Conceptualization: SE; methodology: SE, EK, YL, OL, KS; formal analysis and investigation: SE, EK, KS; writing - original draft preparation: SE, EK; writing - review and editing: SE, EK, YL, OL, KS; funding acquisition: SE; resources: SE, KS; supervision: SE.

Funding Financial support was granted by the German Research Foundation-DFG (ER 786/1-1 to S.E.). Open Access funding enabled and organized by the German DEAL agreement.

Data availability Raw data are available within the supplementary material.

\section{Compliance with ethical standards}

Conflict of interest The authors have no conflicts of interest to declare that are relevant to the content of this article.

Ethics approval Endangered or protected species were not used in this study. Experiments and observations conform to the laws of Germany in relation to animal protection. No specific ethics certification was required for this research.

Consent to participate Not applicable. 
Consent for publication Not applicable.

Open Access This article is licensed under a Creative Commons Attribution 4.0 International License, which permits use, sharing, adaptation, distribution and reproduction in any medium or format, as long as you give appropriate credit to the original author(s) and the source, provide a link to the Creative Commons licence, and indicate if changes were made. The images or other third party material in this article are included in the article's Creative Commons licence, unless indicated otherwise in a credit line to the material. If material is not included in the article's Creative Commons licence and your intended use is not permitted by statutory regulation or exceeds the permitted use, you will need to obtain permission directly from the copyright holder. To view a copy of this licence, visit http://creativecommons.org/licenses/by/4.0/.

\section{References}

Bailey L (1956) Aetiology of European foulbrood; a disease of the larval honey-bee. Nature 178:1030. https://doi.org/10.1038/17811 $30 \mathrm{a} 0$

Bailey L (1959) An improved method for the isolation of Streptococcus pluton, and observations on its distribution and ecology. J Insect Pathol 1:80-85

Bailey L (1960) The epizootiology of European foulbrood of the larval honey bee, Apis mellifera L. J Insect Pathol 2:67-83

Bailey L (1963) The pathogenicity for honey-bee larvae of microorganisms associated with European foulbrood. J Insect Pathol 5:198-205

Blomquist GJ, Bagnères AG (2010) Insect hydrocarbons. Biology, biochemistry, and chemical ecology. Cambridge University Press, USA

Blum MS, Fales HM, Morse RA, Underwood BA (2000) Chemical characters of two related species of giant honeybees (Apis dorsata and A. laboriosa): Possible ecological significance. J Chem Ecol 26:801-807. https://doi.org/10.1023/A:1005476405192

Bolstad BM (2016) PreprocessCore: A collection of pre-processing functions. R package. V 1.36.0.

Breed D, Cook C, McCreery H, Rodriguez M (2015) Recognition in eusocial insects: the honeybee as a model system. In: Aquiloni L, Tricarico E (eds) Social recognition in invertebrates. Springer International Publishing, Switzerland, pp 147-164

Budge GE, Barrett B, Jones B, Pietravalle S, Marris G, Chantawannakul P, Thwaites R, Hall J, Cuthbertson AGS, Brown MA (2010) The occurrence of Melissococcus plutonius in healthy colonies of Apis mellifera and the efficacy of European foulbrood control measures. J Invertebr Pathol 105:164-170. https://doi. org/10.1016/j.jip.2010.06.004

Budge G, Shirley M, Jones B, Quill E, Tomkies V, Feil EJ, Brown MA, Haynes EG (2014) Molecular epidemiology and population structure of the honey bee brood pathogen Melissococcus plutonius. ISME J 8:1588-1597. https://doi.org/10.1038/ismej.2014.20

Crailsheim K, Brodschneider R, Aupinel P, Behrens D, Genersch E, Vollmann J, Riessberger-Gallé U (2013) Standard methods for artificial rearing of Apis mellifera larvae. J Apicult Res 52:1-16. https://doi.org/10.3896/IBRA.1.52.1.05

Cremer S, Armitage SAO, Schmid-Hempel P (2007) Social Immunity. Curr Biol 17:693-702. https://doi.org/10.1016/j.cub.2007.06.008

Djukic M, Erler S, Leimbach A, Grossar D, Charrière J-D, Gauthier L, Hartken D, Dietrich S, Nacke H, Daniel R, Poehlein A (2018) Comparative genomics and description of putative virulence factors of Melissococcus plutonius, the causative agent of
European foulbrood disease in honey bees. Genes 9:419. https:// doi.org/10.3390/genes9080419

Ellis JD, Munn PA (2005) The worldwide health status of honey bees. Bee World 86:88-101. https://doi.org/10.1080/00057 72X.2005.11417323

Erler S, Lewkowski O, Poehlein A, Forsgren E (2018) The curious case of Achromobacter eurydice, a Gram-variable pleomorphic bacterium associated with European foulbrood disease in honeybees. Microb Ecol 75:1-6. https://doi.org/10.1007/s00248-017-1007-x

Evans JD, Spivak M (2010) Socialized medicine: Individual and communal disease barriers in honey bees. J Invertebr Pathol 103:6272. https://doi.org/10.1016/j.jip.2009.06.019

Forsgren E (2010) European foulbrood in honey bees. J Invertebr Pathol 103:5-9. https://doi.org/10.1016/j.jip.2009.06.016

Forsgren E, Budge G, Charrière J, Hornitzky M (2013) Standard methods for European foulbrood research. J Apicult Res 52:1-14. https ://doi.org/10.3896/IBRA.1.52.1.12

Forsgren E, Locke B, Sircoulomb F, Schäfer MO (2018) Bacterial diseases in honeybees. Curr Clin Micro Rpt 5:18-25. https://doi. org/10.1007/s40588-018-0083-0

Gramacho KP, Spivak M (2003) Differences in olfactory sensitivity and behavioral responses among honey bees bred for hygienic behavior. Behav Ecol Sociobiol 54:472-479. https://doi.org/10.1007/ s00265-003-0643-y

Grossar D, Kilchenmann V, Forsgren E, Charrière J-D, Gauthier L, Chapuisat M, Dietemann V (2020) Putative determinants of virulence in Melissococcus plutonius, the bacterial agent causing European foulbrood in honey bees. Virulence 11:554-567. https ://doi.org/10.1080/21505594.2020.1768338

He XJ, Zhang XC, Jiang WJ, Barron AB, Zhang JH, Zeng ZJ (2016) Starving honey bee (Apis mellifera) larvae signal pheromonally to worker bees. Sci Rep 6:22359. https://doi.org/10.1038/srep22359

Le Conte Y, Arnold G, Trouiller J, Masson C, Chappe B, Ourisson G (1989) Attraction of the parasitic mite Varroa to the drone larvae of honeybees by simple aliphatic esters. Science 245:638-639. https://doi.org/10.1126/science.245.4918.638

Le Conte Y, Arnold G, Trouiller J, Masson C (1990) Identification of a brood pheromone on honeybees. Sci Nat 77:334-336. https:// doi.org/10.1007/BF01138390

Le Conte Y, Sreng L, Poitout SH (1995) Brood pheromone can modulate the feeding behavior of Apis mellifera workers (Hymenoptera: Apidae). J Econ Entomol 88:798-804. https://doi.org/10.1093/ jee/88.4.798

Lee ML, Vassilaros DL, White CM, Novotny M (1979) Retention indices for programmed-temperature capillary-column gas chromatography of polycyclic aromatic hydrocarbons. Anal Chem 51:768-773. https://doi.org/10.1021/ac50042a043

Lee S, Lim S, Choi Y-S, Lee M, Kwon HW (2020) Volatile disease markers of American foulbrood-infected larvae in Apis mellifera. J Insect Physiol 122:104040. https://doi.org/10.1016/j.jinsp hys.2020.104040

Lewkowski O, Erler S (2019) Virulence of Melissococcus plutonius and secondary invaders associated with European foulbrood disease of the honey bee. MicrobiologyOpen 8:e649. https://doi. org/10.1002/mbo3.649

Li J, Wang Z, Tan K, Qu Y, Nieh JC (2014) Effects of natural and synthetic alarm pheromone and individual pheromone components on foraging behavior of the giant Asian honey bee, Apis dorsata. J Exp Biol 217:3512-3518. https://doi.org/10.1242/jeb.110171

Maisonnasse A, Lenoir JC, Beslay D, Crauser D, Le Conte Y (2010) E-beta-ocimene, a volatile brood pheromone involved in social regulation in the honey bee colony (Apis mellifera). PLoS ONE 5:e13531. https://doi.org/10.1371/journal.pone.0013531

Martin C, Salvy M, Provost E, Bagnères A, Roux M, Crauser D, Clement J, Le Conte Y (2001) Variations in chemical mimicry by the ectoparasitic mite Varroa jacobsoni according to the 
developmental stage of the host honeybee Apis mellifera. Insect Biochem Molec 31:365-379. https://doi.org/10.1016/S0965 $-1748(00) 00130-2$

Martin C, Provost E, Bagneres AG, Roux M, Clement J, Le Conte Y (2002) Potential mechanism for detection by Apis mellifera of the parasitic mite Varroa destructor inside sealed brood cells. Physiol Entomol 27:175-188. https://doi.org/10.104 6/j.1365-3032.2002.00284.x

McAfee A, Collins TF, Madilao LL, Foster LJ (2017) Odorant cues linked to social immunity induce lateralized antenna stimulation in honey bees (Apis mellifera L.). Sci Rep 7:46171. https://doi. org/10.1038/srep46171

McAfee A, Chapman A, Iovinella I, Gallagher-Kurtzke Y, Collins TF, Higo H, Madilao LL, Pelosi P, Foster LJ (2018) A death pheromone, oleic acid, triggers hygienic behavior in honey bees (Apis mellifera L.). Sci Rep 8:5719. https://doi.org/10.1038/s4159 8-018-24054-2

Mondet F, Kim SH, de Miranda JR, Beslay D, Le Conte Y, Mercer AR (2016) Specific cues associated with honey bee social defence against Varroa destructor infested brood. Sci Rep 6:25444. https ://doi.org/10.1038/srep25444

Nakamura K, Yamazaki Y, Shiraishi A, Kobayashi S, Harada M, Yoshiyama M, Osaki M, Okura M, Takamatsu D (2016) Virulence differences among Melissococcus plutonius strains with different genetic backgrounds in Apis mellifera larvae under an improved experimental condition. Sci Rep 6:33329. https://doi. org/10.1038/srep33329

Nazzi F, Vedova GD, D’Agaro M (2004) A semiochemical from brood cells infested by Varroa destructor triggers hygienic behaviour in Apis mellifera. Apidologie 35:65-70. https://doi.org/10.1051/ apido:2003065

R Core Team (2017) R: a Language and Environment for Statistical Computing. R Foundation for Statistical Computing, Austria

Riessberger-Gallé U, Hernández-López J, Rechberger G, Crailsheim K, Schuehly W (2016) Lysophosphatidylcholine acts in the constitutive immune defence against American foulbrood in adult honeybees. Sci Rep 6:30699. https://doi.org/10.1038/srep30699

Roetschi A, Berthoud H, Kuhn R, Imdorf A (2008) Infection rate based on quantitative real-time PCR of Melissococcus plutonius, the causal agent of European foulbrood, in honeybee colonies before and after apiary sanitation. Apidologie 39:362-371. https://doi. org/10.1051/apido:200819

Salvy M, Martin C, Bagnéres AG, Provost E, Roux M, Le Conte Y, Clement J (2001) Modification of the cuticular hydrocarbon profile of Apis mellifera worker bees in the presence of the ectoparasitic mite Varroa jacobsoni in brood cells. Parasitology 122:145-159. https://doi.org/10.1017/S0031182001007181

Schöning C, Gisder S, Geiselhardt S, Kretschmann I, Bienefeld K, Hilker M, Genersch E (2012) Evidence for damage-dependent hygienic behaviour towards Varroa destructor-parasitised brood in the western honey bee, Apis mellifera. J Exp Biol 215:264-271. https://doi.org/10.1242/jeb.062562

Seidelmann K, Rolke D (2019) Advertisement of unreceptivity-perfume modifications of mason bee females (Osmia bicornis and $O$. cornuta) and a non-existing antiaphrodisiac. PLoS ONE 14:e0215925. https://doi.org/10.1371/journal.pone.0215925

Slessor KN, Winston ML, Le Conte Y (2005) Pheromone communication in the honeybee (Apis mellifera L.). J Chem Ecol 31:27312745. https://doi.org/10.1007/s10886-005-7623-9

Swanson JAI, Torto B, Kells SA, Mesce KA, Tumlinson JH, Spivak M (2009) Odorants that induce hygienic behavior in honeybees: identification of volatile compounds in chalkbrood-infected honeybee larvae. J Chem Ecol 35:1108-1116. https://doi.org/10.1007/ s10886-009-9683-8

Tarr HLA (1937) Studies on European foul brood of bees III further experiments on the production of the disease. Ann Appl Biol 24:614-626. https://doi.org/10.1111/j.1744-7348.1937.tb05857.x

von Büren RS, Oehen B, Kuhn NJ, Erler S (2019) High-resolution maps of Swiss apiaries and their applicability to study spatial distribution of bacterial honey bee brood diseases. PeerJ 7:e6393. https://doi.org/10.7717/peerj.6393

Wagoner K, Spivak M, Hefetz A, Reams T, Rueppell O (2019) Stockspecific chemical brood signals are induced by Varroa and Deformed Wing Virus, and elicit hygienic response in the honey bee. Sci Rep 9:8753. https://doi.org/10.1038/s41598-019-45008-2

Wagoner KM, Millar JG, Schal C, Rueppel O (2020) Cuticular pheromones stimulate hygienic behavior in the honey bee (Apis mellifera). Sci Rep 10:7132. https://doi.org/10.1038/s41598-020-64144 $-8$

Wang Z, Wen P, Qu Y, Dong S, Li J, Tan K, Nieh JC (2016) Bees eavesdrop upon informative and persistent signal compounds in alarm pheromones. Sci Rep 6:25693. https://doi.org/10.1038/ srep25693

White GF (1912) The cause of European foul brood. U.S Department of Agriculture, USA (Circular No. 157)

White GF (1920) European foulbrood. U.S Department of Agriculture, USA (Bulletin No 810)

Wilkins S, Brown MA, Cuthbertson AGS (2007) The incidence of honey bee pests and diseases in England and Wales. Pest Manag Sci 63:1062-1068. https://doi.org/10.1002/ps.1461 